\title{
Vínculos Não-Holônomos e Equações de Voronec
}

\author{
Nonholonomic Constraints and Voronec's Equations
}

Nivaldo A. Lemos

Departamento de Física, Universidade Federal Fluminense, Av. Litorânea s/n, Boa Viagem, 24210-340 Niterói, RJ, Brazil

Recebido em 12 de maio, 2003. Aceito em 09 de junho, 2003.

\begin{abstract}
Será permitido, no contexto do método dos multiplicadores de Lagrange, supor que vínculos não-holônomos já estão em vigor durante a construção da lagrangiana? Este procedimento, embora aplicado com sucesso ao problema da moeda rolante num livro recente, não tem validade geral, como demonstramos por meio de um contra-exemplo. Em muitos casos, o uso de vínculos não-holônomos no processo de construção da lagrangiana é permitido, mas as equações de movimento corretas são as equações de Voronec.

Is it allowed, in the context of the Lagrange multiplier formalism, to assume that nonholonomic constraints are already in effect while setting up Lagrange's function? This procedure, although successfully applied in a recent book to the problem of the rolling penny, is not valid in general, as we show by means of a counterexample. In many cases the use of nonholonomic constraints in the process of construction of the Lagrangian is allowed, but the correct equations of motion are Voronec's equations.
\end{abstract}

No formalismo dos multiplicadores de Lagrange aplicado a sistemas não-holônomos, a lagrangiana é escrita como se não houvesse vínculos. Os vínculos não-holônomos são levados em conta na formulação das equações de movimento, mas não durante a construção da lagrangiana. Construir a lagrangiana admitindo que os vínculos já estão em vigor é completamente equivalente a substituir as equações de vínculo na lagrangiana escrita como se não houvesse vínculos. É tentador admitir como verdadeiro que, juntamente com as equações de vínculo relevantes, a lagrangiana reduzida resultante sempre fornece as equações de movimento corretas do sistema. O procedimento que acabamos de descrever é usado com sucesso num livro recente [1] para resolver o problema de uma moeda rolando sem deslizar sobre um plano inclinado. Infelizmente, ao contrário do que o referido livro parece sugerir, esta abordagem não é válida em geral, como passamos a demonstrar com a ajuda de um contra-exemplo.

Considere uma esfera homogênea rolando sem deslizar num plano horizontal. Este problema é tratado pelo método dos multiplicadores de Lagrange em [2]. Sejam $X, Y, Z$ eixos cartesianos fixos no espaço, com o eixo $Z$ perpendicular ao plano. Os momentos principais de inércia em relação ao centro da esfera são todos iguais a $2 m R^{2} / 5$. Se $x, y$ são as coordenadas do centro da esfera, a lagrangiana, que se confunde com a energia cinética, é dada por

$$
L=\frac{m}{2}\left(\dot{x}^{2}+\dot{y}^{2}\right)+\frac{m R^{2}}{5} \boldsymbol{\omega}^{2} .
$$

As equações de vínculo são

$$
\begin{gathered}
\dot{x}=R \omega_{y}=R \dot{\theta} \operatorname{sen} \phi-R \dot{\psi} \operatorname{sen} \theta \cos \phi, \\
\dot{y}=-R \omega_{x}=-R \dot{\theta} \cos \phi-R \dot{\psi} \operatorname{sen} \theta \operatorname{sen} \phi .
\end{gathered}
$$

Em termos dos ângulos de Euler $\phi, \theta, \psi$ a lagrangiana (1) toma a forma

$$
L=\frac{m}{2}\left(\dot{x}^{2}+\dot{y}^{2}\right)+\frac{m R^{2}}{5}\left(\dot{\phi}^{2}+\dot{\theta}^{2}+\dot{\psi}^{2}+2 \dot{\phi} \dot{\psi} \cos \theta\right)
$$

De acordo com o método empregado em [1], que leva em conta o rolamento sem deslizamento na construção da lagrangiana, a energia cinética é escrita exclusivamente em termos dos graus de liberdade rotacionais tomando os momentos de inércia em relação ao ponto de contato da esfera com o plano. A lagrangiana passa a ser

$$
\bar{L}=\frac{1}{2} \frac{7 m R^{2}}{5}\left(\omega_{x}^{2}+\omega_{y}^{2}\right)+\frac{1}{2} \frac{2 m R^{2}}{5} \omega_{z}^{2} .
$$

Esta é exatamente a lagrangiana que se obtém inserindo as equações de vínculo (2) na lagrangiana (1), que foi escrita como se não houvesse vínculos. Em termos dos ângulos de Euler a lagrangiana reduzida (4) torna-se

$$
\bar{L}=\frac{7 m R^{2}}{10}\left(\dot{\theta}^{2}+\dot{\psi}^{2} \operatorname{sen}^{2} \theta\right)+\frac{m R^{2}}{5}\left(\dot{\phi}^{2}+\dot{\psi}^{2} \cos ^{2} \theta+2 \dot{\phi} \dot{\psi} \cos \theta\right)
$$


Como as variáveis $x$ e $y$ não aparecem na lagrangiana reduzida $\bar{L}$, de acordo com o raciocínio usado em [1] as equações de vínculo (2) não são mais relevantes para a formulação das equações de movimento. Em particular, a equação de Lagrange para $\theta$ é

$$
\frac{d}{d t}\left(\frac{\partial \bar{L}}{\partial \dot{\theta}}\right)-\frac{\partial \bar{L}}{\partial \theta}=0 \Longrightarrow 7 \ddot{\theta}-5 \dot{\psi}^{2} \operatorname{sen} \theta \cos \theta+2 \dot{\phi} \dot{\psi} \operatorname{sen} \theta=0
$$

O tratamento deste problema pelo método dos multiplicadores de Lagrange mostra que os dois multiplicadores de Lagrange são nulos [2]. Segundo a equação (58d) de [2], a equação de movimento correta para $\theta$ é

$$
\ddot{\theta}+\dot{\phi} \dot{\psi} \operatorname{sen} \theta=0
$$

que é completamente diferente da equação (6). As equações diferenciais (6) e (7) em geral fornecem diferentes soluções para $\theta$ porque $\phi, \theta, \psi, \dot{\phi}, \dot{\theta}, \dot{\psi}$ podem ser arbitrariamente escolhidos em qualquer instante particular $t_{0}$. É claro, portanto, que a abordagem sugerida em [1] é desprovida de generalidade, pois funciona para a moeda rolante mas fracassa para a esfera rolante.

Num trabalho anterior [3], observamos que é possível efetuar uma redução da lagrangiana levando em conta os vínculos, mas neste caso as equações de movimento corretas são as equações de Voronec. Dado um sistema dinâmico descrito pela variáveis configuracionais $q_{1}, \ldots, q_{n}$, suponha que as $m$ primeiras velocidades sejam independentes e que as $k=n-m$ velocidades restantes possam ser expressas em termos das demais por meio das equações

$$
\dot{q}_{m+l}-\sum_{j=1}^{m} a_{l j} \dot{q}_{j}=0, l=1, \ldots, k
$$

em que os coeficientes $a_{l j}$ são funções das coordenadas generalizadas $q_{1}, \ldots, q_{n}$.

Seja $L$ a lagrangiana escrita sem levar em consideração as equações de vínculo (8). Se as $k$ últimas velocidades são eliminadas da lagrangiana por meio das equações (8), uma lagrangiana reduzida $\bar{L}$ resulta:

$$
L\left(q_{1}, \ldots, q_{n}, \dot{q}_{1}, \ldots, \dot{q}_{n}, t\right)=\bar{L}\left(q_{1}, \ldots, q_{n}, \dot{q}_{1}, \ldots, \dot{q}_{m}, t\right) .
$$

As equações de movimento de Voronec são [3,4]

$$
\frac{d}{d t}\left(\frac{\partial \bar{L}}{\partial \dot{q}_{i}}\right)-\frac{\partial \bar{L}}{\partial q_{i}}=\sum_{\nu=1}^{k} \frac{\partial \bar{L}}{\partial q_{m+\nu}} a_{\nu i}+\sum_{\nu=1}^{k} \sum_{j=1}^{m} \frac{\partial L}{\partial \dot{q}_{m+\nu}} b_{i j}^{\nu} \dot{q}_{j}, \quad i=1, \ldots, m
$$

em que

$$
b_{i j}^{\nu}=\frac{\partial a_{\nu i}}{\partial q_{j}}-\frac{\partial a_{\nu j}}{\partial q_{i}}+\sum_{\mu=1}^{k}\left(\frac{\partial a_{\nu i}}{\partial q_{m+\mu}} a_{\mu j}-\frac{\partial a_{\nu j}}{\partial q_{m+\mu}} a_{\mu i}\right) .
$$

Fazendo $q_{1}=\phi, q_{2}=\theta, q_{3}=\psi, q_{4}=x, q_{5}=y$, no caso da esfera rolante $m=3$ e $k=2$. As equações de vínculo (2) podem ser escritas na forma (8) com

$$
a_{11}=0, a_{12}=R \operatorname{sen} \phi, a_{13}=-R \operatorname{sen} \theta \cos \phi, a_{21}=0, a_{22}=-R \cos \phi, a_{23}=-R \operatorname{sen} \theta \operatorname{sen} \phi .
$$

A definição (11) fornece imediatamente os únicos coeficientes não nulos $b_{i j}^{\nu}$ :

$$
\begin{gathered}
b_{12}^{1}=-b_{21}^{1}=-R \cos \phi, \quad b_{13}^{1}=-b_{31}^{1}=-R \operatorname{sen} \theta \operatorname{sen} \phi, \quad b_{23}^{1}=-b_{32}^{1}=R \cos \theta \cos \phi, \\
b_{12}^{2}=-b_{21}^{2}=-R \operatorname{sen} \phi, \quad b_{13}^{2}=-b_{31}^{2}=R \operatorname{sen} \theta \cos \phi, \quad b_{23}^{2}=-b_{32}^{2}=R \cos \theta \operatorname{sen} \phi .
\end{gathered}
$$

Segue-se que a equação de Voronec para $\theta$ é

$$
\frac{7 m R^{2}}{5} \ddot{\theta}-m R^{2} \dot{\psi}^{2} \operatorname{sen} \theta \cos \theta+\frac{2 m R^{2}}{5} \dot{\phi} \dot{\psi} \operatorname{sen} \theta=m \dot{x}\left(b_{21}^{1} \dot{\phi}+b_{23}^{1} \dot{\psi}\right)+m \dot{y}\left(b_{21}^{2} \dot{\phi}+b_{23}^{2} \dot{\psi}\right) \text {. }
$$


O uso das equações de vínculo (2) e um pouco de álgebra reduzem esta última equação a

$$
\ddot{\theta}+\dot{\phi} \dot{\psi} \operatorname{sen} \theta=0,
$$

que coincide com a equação correta para $\theta$ fornecida pelo método dos multiplicadores de Lagrange. É igualmente direto verificar que as equações de Voronec restantes para $\phi \mathrm{e}$ $\psi$ coincidem com as obtidas pelo método dos multiplicadores de Lagrange.

Em suma, o método utilizado em [1] não tem validade geral e não deve ser ensinado aos estudantes: o resultado correto obtido para a moeda rolante é fruto de um mero acidente.

Como regra geral, o uso de vínculos não-holônomos na construção da lagrangiana é permitido, mas as equações de movimento corretas são as equações de Voronec, e não as fornecidas pelo método dos multiplicadores de Lagrange.

\section{REFERÊNCIAS}

[1] L. N. Hand e J. D. Finch, Analytical Mechanics (Cambridge University Press, Cambridge, 1998), Sec. 2.8.

[2] E. J. Saletan e A. H. Cromer, Theoretical Mechanics (John Wiley and Sons, New York, 1971), Sec. V-4 (e).

[3] N. A. Lemos, Rev. Bras. Ens. Fís. 25, 28 (2003).

[4] Ju. I. Neĭmark e N. A. Fufaev, Dynamics of Nonholonomic Systems (American Mathematical Society, Providence, Rhode Island, 1972), Cap. III, Sec. 3. 